\title{
Gene Expression Profiling and Functional Characterization of Macrophages in Response to Circulatory Microparticles Produced during Trypanosoma cruzi Infection and Chagas Disease
}

\author{
Imran H. Chowdhury ${ }^{a}$ Sue-jie Koo ${ }^{b}$ Shivali Gupta ${ }^{a}$ Lisa Yi Liang ${ }^{a}$ \\ Bojlul Bahar $^{d}$ Laura Silla $^{a}$ Julio Nuñez-Burgos ${ }^{\mathrm{e}}$ Natalia Barrientos ${ }^{\mathrm{e}}$ \\ Maria Paola Zago ${ }^{f}$ Nisha Jain Garga-c \\ Departments of a Microbiology and Immunology and ${ }^{\mathrm{b}}$ Pathology, and ${ }^{\mathrm{c} I n s t i t u t e}$ for Human Infections and Immunity \\ (IHII) and Sealy Center for Vaccine Development (SCVD), University of Texas Medical Branch (UTMB), Galveston, \\ TX, USA; ${ }^{d}$ International Institute of Nutritional Sciences and Applied Food Safety Studies, University of Central

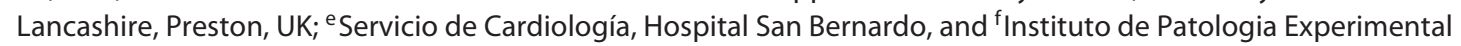 \\ (IPE), Universidad Nacional de Salta y Consejo Nacional de Investigaciones Cientificas y Técnicas (UNSa-CONICET), \\ Salta, Argentina
}

\section{Keywords}

Chagasic cardiomyopathy · Microparticles ·

Metabolic inflammatory gene expression profile .

Macrophage activation

\begin{abstract}
Background: Chronic inflammation and oxidative stress are hallmarks of chagasic cardiomyopathy (CCM). In this study, we determined if microparticles (MPs) generated during Trypanosoma cruzi $(T c)$ infection carry the host's signature of the inflammatory/oxidative state and provide information regarding the progression of clinical disease. Methods: MPs were harvested from supernatants of human peripheral blood mononuclear cells in vitro incubated with Tc (control: LPS treated), plasma of seropositive humans with a clinically asymptomatic (CA) or symptomatic (CS) disease state (vs. normal/healthy $[\mathrm{NH}]$ controls), and plasma of mice immunized with a protective vaccine before challenge infection
\end{abstract}

\section{KARGER}

(C) 2016 S. Karger AG, Basel

E-Mail karger@karger.com

www.karger.com/jin (control: unvaccinated/infected). Macrophages (m $\varphi s$ ) were incubated with MPs, and we probed the gene expression profile using the inflammatory signaling cascade and cytokine/chemokine arrays, phenotypic markers of $m \varphi$ activation by flow cytometry, cytokine profile by means of an ELISA and Bioplex assay, and oxidative/nitrosative stress and mitotoxicity by means of colorimetric and fluorometric assays. Results: Tc- and LPS-induced MPs stimulated proliferation, inflammatory gene expression profile, and nitric oxide ( $\mathrm{NO}$ ) release in human THP-1 mys. LPS-MPs were more immunostimulatory than Tc-MPs. Endothelial cells, T lymphocytes, and $m \varphi s$ were the major source of MPs shed in the plasma of chagasic humans and experimentally infected mice. The CS and CA (vs. NH) MPs elicited >2-fold increase in NO and

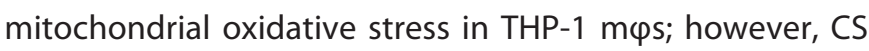

Imran H. Chowdhury, Sue-jie Koo, Shivali Gupta, and Nisha Jain Garg contributed equally to this work. 
(vs. CA) MPs elicited a more pronounced and disease-statespecific inflammatory gene expression profile (IKBKB, NR3C1, and TIRAP vs. CCR4, EGR2, and CCL3), cytokine release (IL-2 $+\mathrm{IFN}-\gamma>\mathrm{GCSF}$ ), and surface markers of $\mathrm{m} \varphi$ activation (CD14 and CD16). The circulatory MPs of nonvaccinated/infected mice induced 7.5 -fold and $40 \%$ increases in $\mathrm{NO}$ and IFN- $\gamma$ production, respectively, while these responses were abolished when RAW264.7 $\mathrm{m} \varphi$ s were incubated with circulatory MPs of vaccinated/infected mice. Conclusion: Circulating MPs reflect in vivo levels of an oxidative, nitrosative, and inflammatory state, and have potential utility in evaluating disease severity and the efficacy of vaccines and drug therapies against CCM.

(c) 2016 S. Karger AG, Basel

\section{Introduction}

Trypanosoma cruzi (TC) is endemic in Latin America and is an emerging infection in the USA [1]. The prevalence of human $T c$ infection is approximately 20 million, and 120 million are at risk of infection in Latin America [1]. Vectors carrying $T c$ are wide-spread in the USA, and the Centers for Disease Control and Prevention estimate that $>300,000$ infected individuals are living in the USA [2]. Unfortunately, exposure to $T c$ remains undetected for several years, when patients then display cardiac insufficiency due to tissue fibrosis, ventricular dilation, and arrhythmia [3]. Chagasic cardiomyopathy (CCM) results in a loss of 2.74 million disability-adjusted life-years, and $>15,000$ deaths due to heart failure per year [1].

Macrophages (mps) are the immune cells that are essential for controlling Tc infection $[4,5]$. It is suggested that $\mathrm{m} \varphi$-derived peroxynitrite, a strong cytotoxic agent that is formed by the reaction of nitric oxide ( $\left.{ }^{\circ} \mathrm{NO}\right)$ with superoxide $\left(\mathrm{O}_{\overline{2}}^{\overline{2}}\right)$, plays a major role in the direct killing of Tc [6]. Infected experimental animals and humans also elicit strong adaptive immunity, constituted of antiparasite lytic antibodies, type 1 cytokines, and antigen-specific cytolytic T lymphocytes [review 7]. These immune responses are capable of keeping the parasite burden under control but lack the ability to achieve pathogen clearance [8], leading to low-level parasite persistence.

In recent years, we and other study groups have shown that $T c$ invasion elicits functional changes in mitochondrial respiratory chain complexes, and this initial insult continues and serves as a major source of the increased production of $\mathrm{O}_{2}^{\overline{2}}$ radicals in the heart [9]. Control of Tcinduced reactive oxygen species (ROS) by using chemical antioxidants or by genetically enhancing the expression of superoxide dismutase $\left(\mathrm{SOD}_{2}\right)$ results in an improvement in cardiac mitochondrial respiratory chain function in chagasic mice $[10,11]$. Importantly, $\mathrm{SOD}_{2}$-overexpressing mice also exhibit a lower degree of inflammatory infiltrates and mitochondrial damage that is otherwise pronounced in chagasic myocardium [12]. These studies suggest that $T c$-induced ROS are not only associated with chronic oxidative stress but may also signal the activation and recruitment of inflammatory infiltrate in the chagasic heart.

The role of ROS in signaling inflammatory immune responses in Chagas disease is not completely understood. Extensive infiltration of gp $91^{\text {phox }}$ (NOX2 component) $m \varphi$ clusters associated with oxidative adducts has been noted in chagasic hearts $[13,14]$. Macrophages in vitro incubated with heart homogenates or the plasma of infected mice were found to elicit a proinflammatory response, demonstrated by an increased production of ROS, $N O$, and TNF- $\alpha$ [15]. Heart homogenates of chagasic mice or of normal mice in vitro, oxidized with $\mathrm{H}_{2} \mathrm{O}_{2}$ or peroxynitrite, are recognized by antibodies present in the sera of the $T c$-infected host [15]. These studies suggest that oxidative stress-induced adducts are potentially responsible for the chronic activation of inflammatory $m \varphi s$ and non- $T c$-specific antibody response in Chagas disease. The practical and ethical limitations in obtaining cardiac biopsies, however, prohibit using ROS production and ROS-induced antigen generation and $m \varphi$ activation as early indicators for identifying patients at risk of developing clinically symptomatic CCM.

Microparticles (MPs) are small vesicles harboring ligands, receptors, active lipids, or RNA/DNA from their cell of origin [16]. In pathological conditions, a stimulus that triggers MP formation regulates the selective sorting of constituents and the composition of MPs, and, consequently, the biological information that they transfer [17]. Thus, MPs can play roles in intercellular communication and be able to modulate important cellular regulatory functions. There is no literature or information in the public domain on the potential effects of MPs on the molecular mechanisms implicated in the pathophysiology of CCM.

In this study, we aimed to determine whether circulating MPs generated during Tc infection carry the host's signature of inflammatory/oxidative pathology and provide information regarding clinical disease severity. We isolated MPs released in the (1) supernatants of human peripheral blood mononuclear cells in vitro infected with $T c$ and (2) plasma of human subjects who were characterized as seropositive with clinically asymptomatic (CA) or 
symptomatic (CS) Chagas disease. We then employed high-throughput transcriptomic and physiological approaches to study the $\mathrm{m} \varphi$ response to MPs. We also used MPs from a mouse model of vaccination and chronic disease to determine if $m \varphi$ response to circulating MPs provides an indication of cure from infection.

\section{Materials and Methods}

\section{Ethics Statement}

All animal experiments were performed according to the National Institutes of Health Guide for the Care and Use of Experimental Animals, and approved by the Institutional Animal Care and Use Committee (IACUC) at the University of Texas Medical Branch (UTMB), Galveston (protocol No. 0805029).

The collection of human peripheral blood samples was approved by the institutional review board at the UTMB, Galveston (protocol No. IRB13-0367) and the ethics committee at the Universidad Nacional de Salta, Salta, Argentina. A written informed consent was obtained from all individuals visiting the Cardiologic Unit of San Bernardo Hospital in Salta Argentina for clinical service. The leftover blood samples collected for clinical purpose were decoded and deidentified before they were provided for research purposes.

\section{Human Samples}

The Tc-specific antibodies were analyzed in all sera samples by using the Chagatest ELISA recombinant (v4.0) and Chagatest HAI kits (Wiener, Rosario, Argentina). Sera samples were considered seropositive if both tests identified the presence of anti- $T c$ antibodies. Electrocardiography (ECG, 12-lead at rest and 3-lead with exercise) and transthoracic echocardiography were performed for evaluating the heart function in all individuals. Normal healthy $(\mathrm{NH}, n=10)$ controls were seronegative and exhibited no history or clinical symptoms of heart disease. Seropositive individuals were grouped as CA $(n=10)$ when they exhibited none-to-minor ECG abnormalities, no left ventricular dilatations, and normal ejection fraction of 55-70\%. Seropositive individuals were categorized as CS $(n=10)$ when they displayed a varying degree of ECG abnormalities, systolic dysfunction (ejection fraction $<55 \%$ ), left ventricular dilatation (diastolic diameter $\geq 57 \mathrm{~mm}$ ), and/or potential signs of heart failure [18].

\section{Mice, Immunization, and Challenge Infection}

All chemicals used in the study were of molecular grade, and purchased from Sigma-Aldrich (St. Louis, MO, USA) unless otherwise specified. C57BL/6 female mice (wild type) were purchased from Harlan Laboratories (Indianapolis, IN, USA). Tc (Sylvio$\mathrm{X} 10 / 4$ ) and $\mathrm{C} 2 \mathrm{C} 12$ cells (an immortalized, mouse-derived myoblast cell line) were purchased from the American Type Culture Collection (ATCC, Manassas, VA, USA) and Tc trypomastigotes (infective stage) were propagated in $\mathrm{C} 2 \mathrm{C} 12$ cells. The $T c$ antigens TcG2 and TcG4 were used as vaccine candidates and have been described in detail previously $[19,20]$. Mice ( $n=5$ /group/experiment; 2 experiments) were injected in the quadriceps muscle with TcG2 and TcG4 antigens that were delivered as a DNA-prime/ protein-boost vaccine $[19,20]$. Two weeks after immunization, they were challenged with Tc trypomastigotes (10,000/mouse), and sacrificed at approximately 120 days after infection, corresponding to the chronic disease phase $[19,20]$. Plasma samples were subjected to isolation of circulating MPs (described below). Protein levels were determined by using the Bradford protein assay (Bio-Rad, Hercules, CA, USA).

\section{MP Isolation}

Human blood samples were drawn in EDTA-containing Vacutainer cell preparation tubes (BD Biosciences, San José, CA, USA). The tubes were centrifuged for $20 \mathrm{~min}$ each at 270 and $1,000 \mathrm{~g}$ to separate plasma. Plasma samples were subjected to 3 series of centrifugation at $15,000 \mathrm{~g}$ for $15 \mathrm{~min}$ each, and pelleted MPs were washed with RPMI media and stored at $-80^{\circ} \mathrm{C}$. A similar protocol was followed for isolating the MPs from murine plasma samples.

In some experiments, buffy coat obtained after separation of plasma was subjected to Ficoll Hypaque ${ }^{\mathrm{TM}}$ density gradient (GE Healthcare, Pittsburgh, PA, USA), and centrifuged at $400 \mathrm{~g}$ for 30 min. The enriched human peripheral blood mononuclear cell (PBMC) pellets were washed with RPMI media, seeded in 24-well plates $\left(0.5-1 \times 10^{6}\right.$ cells $/$ well $\left./ \mathrm{mL}\right)$, and incubated in triplicate with Tc (cell-to-parasite ratio, 1:3) or LPS (100 ng/mL) in RPMI me$\mathrm{dia} / 10 \% \mathrm{FBS}$ media at $37^{\circ} \mathrm{C} / 5 \% \mathrm{CO}_{2}$ for $48 \mathrm{~h}$. MPs from the supernatants were collected as above.

\section{Treatment of Macrophages with MPs}

THP-1 human monocytes (ATCC TIB-202) were suspended in complete RPMI media and incubated at $37^{\circ} \mathrm{C} / 5 \% \mathrm{CO}_{2}$ for $24 \mathrm{~h}$ in the presence of $50 \mathrm{ng} / \mathrm{mL}$ phorbol-12-myristate-13-acetate (PMA, Sigma-Aldrich), and then for $48 \mathrm{~h}$ in complete RPMI media without any stimulus to generate the resting $\mathrm{m} \varphi \mathrm{s}$ [21]. RAW264.7 murine $\mathrm{m} \varphi \mathrm{s}$ (ATCC TIB-71) were cultured in high-glucose Dulbecco's modified Eagle's medium (DMEM) containing 10\% fetal bovine serum (Invitrogen, Carlsbad, CA, USA), $2 \mathrm{mmol} / \mathrm{L}$ glutamine, $100 \mathrm{IU} / \mathrm{mL}$ penicillin, and $100 \mu \mathrm{g} / \mathrm{mL}$ streptomycin (Corning, Corning, NY, USA). THP-1 (human) or RAW264.7 (murine) m $\varphi s$ were seeded in 6 -well $\left(1 \times 10^{6}\right.$ cells/well $), 24$-well $\left(5 \times 10^{5}\right.$ cells/ well $)$, or 96 -well $\left(1 \times 10^{4}\right.$ cells/well $)$ plates, or in Nunc Lab-Tek II chamber slides $\left(1 \times 10^{4}\right.$ cells/well, Thermo Scientific, Waltham, MA, USA) and incubated for $2 \mathrm{~h}$ to allow the cells to adhere. Serum-free media was added, and $m \varphi s$ were incubated in triplicate with MPs isolated from human or mouse plasma (10\% plasma equivalent) or from media of Tc-infected cells ( $10 \%$ media equivalent). Macrophages ( \pm MPs) were incubated for $1,12,24$, or $48 \mathrm{~h}$ and cells and supernatants were stored at $-80^{\circ} \mathrm{C}$.

\section{Gene Expression Profiling by Real-Time RT-qPCR}

The quantitative expression profiling of a panel of 91 human genes involved in the inflammatory signaling cascade was performed by using custom-designed arrays printed by Sigma-Aldrich. Full details of the arrays were previously described [22]. Gene expression profiling for human cytokines/chemokines was performed by using an in-house PCR array that consisted of 89 genes [23]. All primer sequences are available upon request.

THP- 1 m $\varphi$ s were seeded in 12 -well plates $\left(1 \times 10^{6}\right.$ cells/well $)$ and incubated in triplicate with MPs for $12 \mathrm{~h}$. Cells were suspended in TRIzol reagent, and total RNA was extracted and precipitated by chloroform/isopropanol/ethanol method. DNA that could contaminate the RNA preparation was removed by deoxyribonuclease I (DNase I) treatment (Ambion, Austin, TX, USA). 
Total RNA absorbance at 260 and $280 \mathrm{~nm}$ was read by using a NanoDrop ND-1000 spectrophotometer (Wilmington, DE, USA) to assess the quality $\left(\mathrm{OD}_{260 / 280}\right.$ ratio $\left.>2.0\right)$ and quantity $\left(\mathrm{OD}_{260}\right.$ of $1=40 \mu \mathrm{g} / \mathrm{mL}$ RNA). First-strand cDNA was synthesized from DNaseI-treated 1- $\mu \mathrm{g}$ RNA sample using the iScript ${ }^{\mathrm{TM}} \mathrm{cDNA}$ synthesis kit (Bio-Rad) and diluted 5-fold with nuclease-free $\mathrm{ddH}_{2} \mathrm{O}$. Quantitative real-time PCR was performed in a $20-\mu \mathrm{L}$ reaction containing $1 \mu \mathrm{L}$ cDNA, $10 \mu \mathrm{L}$ SYBR Green master mix (Bio-Rad), and $500 \mathrm{nM}$ of each gene-specific oligonucleotide. The thermal cycle conditions were $94^{\circ} \mathrm{C}$ for $30 \mathrm{~s}$ followed by $60^{\circ} \mathrm{C}$ for $1 \mathrm{~min}$, for 40 cycles. The PCR Base Line Subtracted Curve Fit mode was applied for determining the threshold cycle, $C_{t}$, using iCycler iQ realtime detection system software (Bio-Rad). For each target gene, $\mathrm{C}_{t}$ values were normalized to the $\mathrm{C}_{\mathrm{t}}$ values for the $\beta$-actin $(A C T B)$ and $\beta$-glucuronidase (GUSB) reference genes. The relative expression level of each target gene was calculated according to the $2^{-\Delta \Delta C_{t}}$ method, where $\Delta C_{t}$ represents the $C_{t}$ (target) $-C_{t}$ (reference), and $\Delta \Delta \mathrm{C}_{\mathrm{t}}$ represents $\Delta \mathrm{C}_{\mathrm{t}}$ (sample) $-\Delta \mathrm{C}_{\mathrm{t}}$ (no treatment control or control MP treatment) [21].

\section{Cell Viability}

THP-1 m $\varphi$ s were seeded to 96 -well plates $\left(1 \times 10^{4}\right.$ cells $/ 200 \mu \mathrm{L} /$ well), and incubated in triplicate with serum-free media ( \pm MPs) for $24 \mathrm{~h}$. Cells were loaded with 10\% (v/v) AlamarBlue (Life Technologies, Carlsbad, CA, USA) during the last $3 \mathrm{~h}$ of incubation. AlamarBlue metabolism by functional mitochondria, resulting in cleavage of resazurin into fluorescent resorufin $\left(\mathrm{Ex}_{560 \mathrm{~nm}} / \mathrm{Em}_{590 \mathrm{~nm}}\right)$ was recorded by using a SpectraMax M5 spectrophotometer (Molecular Devices, Sunnyvale, CA, USA).

\section{ROS and $\cdot$ NO Levels}

Reactive oxygen species (ROS) were monitored by using $2^{\prime}, 7^{\prime}$ dichlorodihydrofluorescein diacetate $\left(\mathrm{H}_{2} \mathrm{DCFDA}\right.$, Invitrogen) fluorescent probe. Briefly, THP-1 m $\varphi$ s were seeded in 96-well plates $\left(1 \times 10^{4}\right.$ cells per well $)$, were allowed to adhere for $2 \mathrm{~h}$, and were then incubated for $1 \mathrm{~h}$ in triplicate with MPs. Supernatants were harvested, and cells were washed and loaded with $10 \mu \mathrm{M}$ of $\mathrm{H}_{2}$ DCFDA in $100 \mu \mathrm{L}$ of serum-free media, and incubated in the dark for $30 \mathrm{~min}$ at $37^{\circ} \mathrm{C}$. Cells were washed 3 times with phenolred-free media and $\mathrm{H}_{2}$ DCFDA oxidation by intracellular ROS, resulting in the formation of fluorescent dichlorodihydrofluorescein (DCF, $\mathrm{Ex}_{498 \mathrm{~nm}} / \mathrm{Em}_{598 \mathrm{~nm}}$ ), was recorded by fluorimetry. Cells treated with $0.1-1 \mu \mathrm{M} \mathrm{H}_{2} \mathrm{O}_{2}$ were used as a positive control.

The ${ }^{\circ} \mathrm{NO}$ level (an indicator of inducible ${ }^{*} \mathrm{NO}$ synthase activity) was monitored by Griess reagent assay. Briefly, samples were reduced with 0.01 unit $/ 100 \mu \mathrm{L}$ of nitrate reductase, and incubated for 10 min with $100 \mu \mathrm{L}$ of $1 \%$ sulfanilamide made in $5 \%$ phosphoric acid/0.1\% N-(1-napthyl) ethylenediamine dihydrochloride (1:1, $\mathrm{v} / \mathrm{v})$. Formation of diazonium salt was monitored at $545 \mathrm{~nm}$ by spectrophotometry (standard curve: $2-50 \mu \mathrm{M}$ sodium nitrite) [24].

\section{Mitochondrial Membrane Potential and ROS}

To examine the changes in mitochondrial membrane potential, $\mathrm{m} \varphi \mathrm{s}$ were seeded and MPs were added in triplicate. Macrophages were incubated for $24 \mathrm{~h}$ with the MPs, washed, and then with $10 \mu \mathrm{M} 5,5^{\prime}, 6,6^{\prime}$-tetrachloro-1,1',3,3'-tetraethylbenzimidazolylcarbocyanine iodide (JC-1, Molecular Probes, Eugene, OR, USA) for $30 \mathrm{~min}$. Cells were washed twice with cold PBS to remove the excess dye, suspended in serum-free/phenol-red-free RPMI, and fluorescence was measured as above. JC-1 dye in respiring mito- chondria is converted from green $\left(\mathrm{Ex}_{485 \mathrm{~nm}} / \mathrm{Em}_{530 \mathrm{~nm}}\right)$ to red $\left(\mathrm{Ex}_{530 \mathrm{~nm}} / \mathrm{Em}_{580 \mathrm{~nm}}\right)$ fluorescent J-aggregates and provides a sensitive in dication of the changes in mitochondrial membrane potential $(\Delta \Psi \mathrm{m})$.

To measure mitochondrial ROS production, $\operatorname{m} \varphi s$ ( \pm MPs) were incubated in the dark for 30 min with $5 \mu \mathrm{M}$ MitoSOX Red (Invitrogen). MitoSOX Red oxidation by mitochondrial ROS, resulting in red fluorescence $\left(\mathrm{Ex}_{518 \mathrm{~nm}} / \mathrm{Em}_{605 \mathrm{~nm}}\right)$, was detected by fluorimetry.

\section{Cytokine Levels}

A Bio-Plex Pro Human Cytokine 17-plex assay (Bio-Rad M5000031YV) was employed to profile the concentration of human cytokines and chemokines. Briefly, THP-1 m $\varphi$ s were seeded in 48-well plates in $500 \mu \mathrm{L}$ media and MPs were added in triplicate. After incubation for $24 \mathrm{~h}$, culture supernatants $(50 \mu \mathrm{L})$ were transferred in duplicate to the plates precoated with cytokine-specific antibodies conjugated with different color-coded beads, and the plates were incubated for $1 \mathrm{~h}$, washed, and then sequentially incubated with $50 \mu \mathrm{L}$ of biotinylated cytokine-specific detection antibodies and streptavidin-phycoerythrin conjugate. Fluorescence was recorded using a SpectraMax M5 microplate reader, and cytokine/chemokine concentrations were calculated with Bio-Plex Manager software (v5) by using a standard curve derived from recombinant cytokines $(2-32,000 \mathrm{pg} / \mathrm{mL})$.

In some experiments, THP- $1 \mathrm{~m} \varphi$ s seeded in 24 -well plates $(5 \times$ $10^{5}$ cells $/$ well $/ \mathrm{mL}$ ) were incubated in triplicate with human MPs for $48 \mathrm{~h}$. Culture supernatants were utilized for the measurement of cytokine release (IL- $1 \beta$, IL-4, IL-10, IFN- $\gamma$, and TNF- $\alpha$ ) using human cytokine optEIA ${ }^{\mathrm{TM}}$ ELISA kits (Pharmingen, San Diego, CA, USA). Likewise, supernatants from RAW264.7 m $\varphi$ s incubated for $48 \mathrm{~h}$ with MPs isolated from normal, chagasic and vaccinated/ chagasic mice were analyzed for IL- $1 \beta$, IL- 4 , IL-10, IFN- $\gamma$, and TNF- $\alpha$ levels by using murine cytokine optEIA ${ }^{\mathrm{TM}}$ ELISA kits (Pharmingen).

\section{Flow Cytometry}

To evaluate changes in the expression of surface markers in response to MPs, THP-1 m $\varphi$ s were seeded in 6-well plates $\left(1 \times 10^{6}\right.$ cells/well $/ \mathrm{mL}$ ), and incubated in triplicate with MPs (10\% serum equivalent) for $24 \mathrm{~h}$. Cells were harvested, pelleted and suspended in $50 \mu \mathrm{L}$ of stain buffer (PBS with $2 \%$ FBS). Suspended cells were stained for 30 min with antibody cocktails containing human peridinin chlorophyll protein (PerCP)-anti-CD14, allophycocyanin (APC-Cy7)-anti-CD16, APC-anti-CD206, phycoerythrin (PECy7)-anti-CD64, PE-Cy5-anti-CD80, and V-450-anti-CD200 fluorescence-conjugated antibodies (BD Biosciences, Franklin Lakes, NJ, USA). The stained cells were washed, fixed with $2 \%$ paraformaldehyde, and then analyzed by 6 -color flow cytometry on an LSRII Fortessa cell analyzer. Cells stained with isotype-matched IgGs were used as controls. Macrophages were gated based on parameters of forward and side light scatter and data acquisition was performed on a minimum of 10,000-gated events. Data were analyzed using FlowJo software (v7.6.5, TreeStar, San Carlo, CA, USA). The mean fluorescence intensity was derived from fluorescence histograms, and was adjusted for background with isotypematched controls.

To evaluate the cellular origin of the MPs, these were isolated from the media of human PBMCs in vitro infected with Tc and from the plasma of clinically characterized human subjects as de- 
Fig. 1. Macrophage gene expression profile response to MPs released by T. cruzi (Tc)infected cells. Human PBMCs were incubated for $48 \mathrm{~h}$ with media alone, LPS or $T c$, and supernatants were centrifuged to harvest the control (Con-MPs), LPS-induced (LPS-MPs), and Tc-induced MPs (TcMPs) MPs. THP-1 m $\varphi$ s were incubated in triplicate with MPs for $12 \mathrm{~h}$. Total RNA from each sample was reverse transcribed, and CDNA was used for real-time PCR with the inflammation signaling cascade and cytokine/chemokine arrays. The differential mRNA level was normalized to housekeeping genes, and the fold change in gene expression was calculated (online suppl. Table S1). a Venn diagram of comparative analysis of gene expression profile $(\geq|1.5|$ fold change) induced by LPS-MPs and TcMPs in comparison to that noted in ConMP-treated $m \varphi s$. b Mean differential expression of top molecules $(\geq|2.0|$ fold change) induced by LPS-MPs and Tc-MPs (vs. Con-MPs) in THP-1 m $\varphi s$.

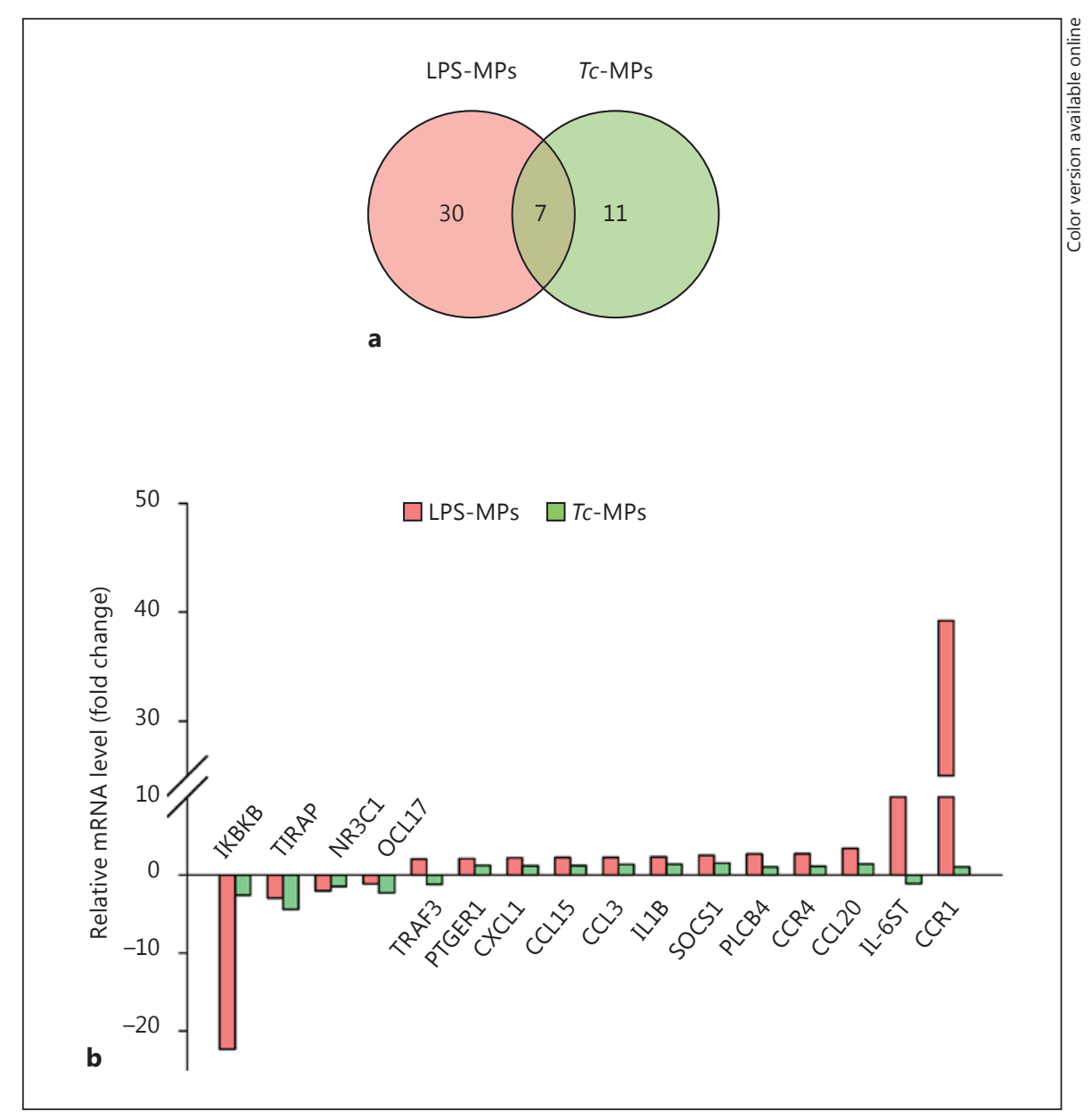

scribed above. The MPs were also isolated from the plasma of normal and experimentally infected mice. They were resuspended in annexin $\mathrm{V}$ binding buffer and labeled for $30 \mathrm{~min}$ on ice with APCor FITC-conjugated anti-annexin V antibody. Simultaneously, MPs were labeled with mouse or human PerCP-anti-CD14, APC-Cy7-anti-CD61, PE-Cy7-anti-CD62E, V-450-anti-troponin, FITC-anti-CD4, and PE-anti-CD8 fluorescence-conjugated antibodies (5-10 $\mu \mathrm{L} /$ sample, e-Biosciences). Stained MPs were washed with cold PBS, and flow cytometry was performed on an LSRII Fortessa cell analyzer. In order to separate true events from background noise and unspecific binding of antibodies to debris, we defined MPs as particles that were $<1 \mu \mathrm{m}$ in diameter and had positive staining for annexin $\mathrm{V}$.

\section{Statistical Analysis}

All in vitro and in vivo experiments were repeated at least twice, and conducted with triplicate observations per sample, and data are expressed as mean \pm SEM. All data were analyzed using InStat v3 (GraphPad, La Jolla, CA, USA). Data were analyzed by the Student $t$ test (for comparison of 2 groups) and one-way analysis of variance (ANOVA) with the Tukey post hoc test (for comparison of multiple groups). Significance is presented as $* p<0.05,{ }^{* *} p<$ 0.01 , and $* * * p<0.001$.

MPs in Chagas Disease and Cure

\section{Results}

We first utilized an in vitro system to determine if $T c$ infection produces MPs capable of activating m $\varphi s$. For this, human PBMCs were incubated for $48 \mathrm{~h}$ with $T c$ and supernatants were centrifuged to harvest the $T c$-induced MPs (Tc-MPs). PBMCs were also incubated with LPS or media alone for $48 \mathrm{~h}$, and supernatants were centrifuged to harvest the LPS-induced MPs (LPS-MPs) and nontreated/control MPs (Con-MPs), respectively. We incubated THP- $1 \mathrm{~m} \varphi \mathrm{s}$ for $12 \mathrm{~h}$ with MPs, and first performed qRT-PCR analysis using the inflammatory signaling cascade and cytokine/chemokine arrays to probe the expression of 180 genes (including the housekeeping genes). The data were normalized to housekeeping genes, and the relative change in gene expression in THP-1 m $\mathrm{ms}$ incubated with sample MPs (vs. Con-MPs) was calculated. These data showed that 37 genes (30 upregulated, 7 downregulated) and 18 genes (5 upregulated, 13 downregulated) were differentially expressed $(\geq|1.5|$ fold 
Fig. 2. Functional response of $\mathrm{m} \varphi \mathrm{s}$ incubated with Tc-MPs. THP-1 m $\varphi$ s were incubated in triplicate with medium only (none) or with Con-MPs, LPS-MPs, and Tc-MPs for $1 \mathrm{~h}$. Supernatants were utilized to measure ROS release by amplex red assay (a) and $\mathrm{NO}$ production by Griess reagent assay (b). c THP-1 m $\varphi$ s were loaded with a JC-1 probe, and change in mitochondrial membrane potential was measured as J aggregates (red)/J monomers (green) ratio by fluorimetry. d THP-1 m $\varphi$ s were incubated with MPs or media alone for $21 \mathrm{~h}$, and then loaded with AlamarBlue for $3 \mathrm{~h}$. The cell proliferation and viability was determined by resorufin fluorescence. Data are shown as mean value \pm SEM, and significance is presented as $* p<0.05,{ }^{* *} p<0.01,{ }^{* * *} p<$ 0.001 (no treatment vs. MP treatment).

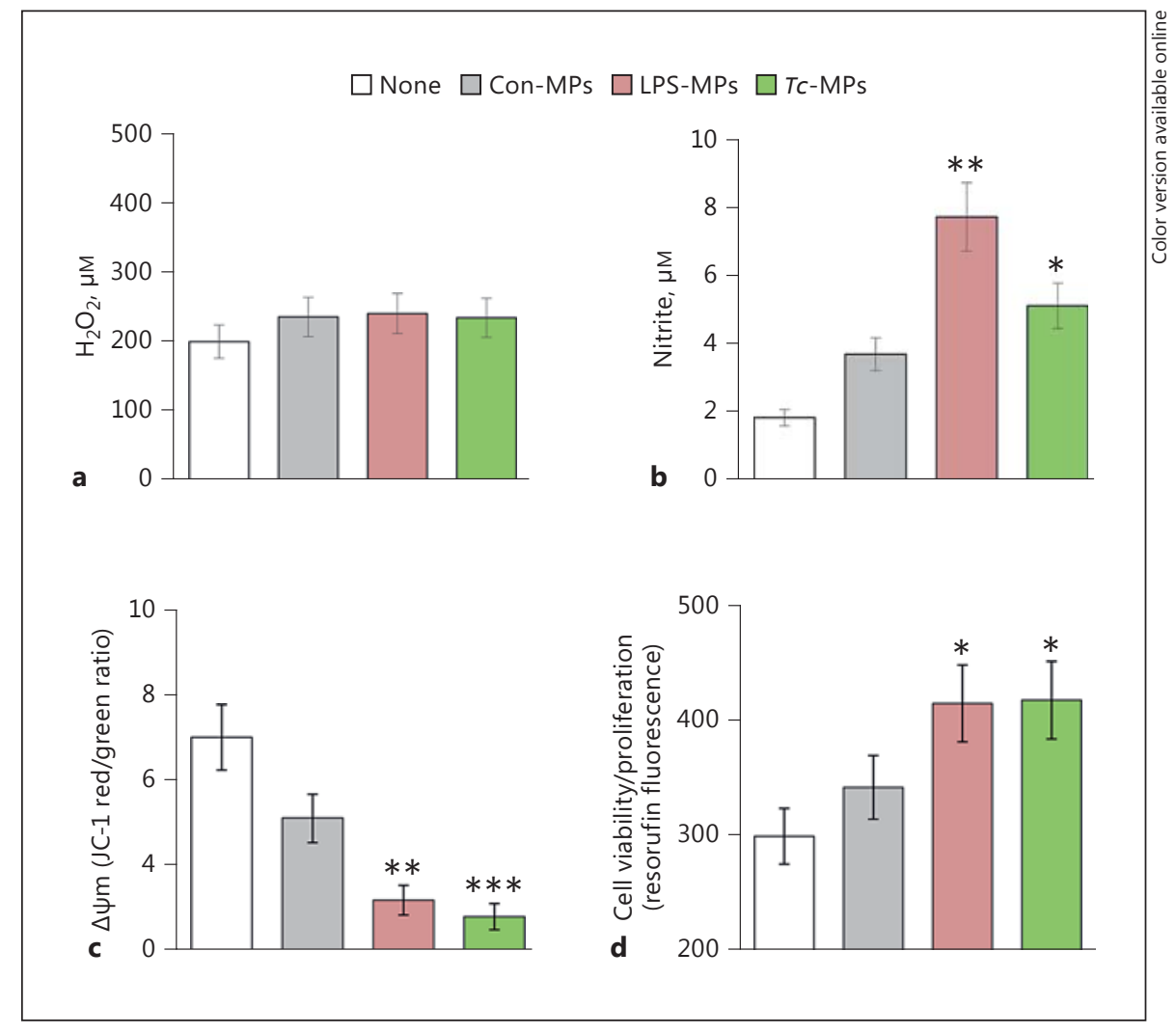

change, $p<0.05)$ in THP-1 m $\varphi$ s incubated with LPS-MPs and Tc-MPs, respectively, when compared to that noted in $\mathrm{m} \varphi \mathrm{s}$ incubated with Con-MPs (online suppl. Table S1; for all online suppl. material, see www. karger.com/doi/10.1159/000451055). Of these, 7 genes were differentially expressed ( $\downarrow N R 3 C 1, \uparrow S O C S 1, \uparrow C X C L 5, \uparrow I L-10)$ by both LPS-MPs

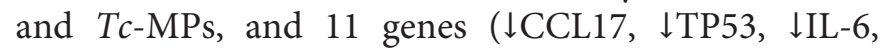
$\downarrow$ EGR2, NR2C2, EGFR, $\downarrow$ PTGER2, TNFSF18, INSR, $\uparrow I L-4, \uparrow I L-2 R A)$ were differentially expressed in $m \varphi s$ in a Tc-MPs-specific manner (Fig. 1a; online suppl. Table S1). The top molecules that were differentially expressed $>2$ fold in THP- $1 \mathrm{~m} \varphi$ s by LPS-MPs and Tc-MPs in comparison to Con-MPs are shown in Figure $1 \mathrm{~b}$.

To assess if $T c$-MPs elicited a functional response in immune cells, we incubated the THP-1 m $\varphi$ s with MPs for $1 \mathrm{~h}$, and examined the oxidative/nitrosative response and mitochondrial stress levels. No increase in $\mathrm{H}_{2} \mathrm{O}_{2}$ release (amplex red assay) was induced by LPS-MPs and Tc-MPs when compared to that noted in $m \varphi s$ incubated in media alone or with Con-MPs (Fig. 2a). Macrophages incubated with LPS-MPs and Tc-MPs exhibited a 3.2-fold and 1.7fold increase in nitrite release, respectively, compared to
THP-1 m $\varphi s$ incubated in media alone (Fig. $2 b, p<0.05$ ). JC-1 forms J-aggregates (red) in mitochondria and JC-1 red/green ratio provides a sensitive indicator of cellular stress. The LPS-MPs and Tc-MPs elicited a 56 and $60 \%$ decline in JC-1 red/green ratio, respectively (Fig. $2 c, p<0.01$ ). Macrophage activation is followed by cell proliferation. Resazurin (alamarBlue) metabolism to fluorescent resorufin by mitochondrial aerobic respiration provides a sensitive measure of cell viability and proliferation. THP-1 $\mathrm{m} \varphi s$ incubated with LPS-MPs and Tc-MPs for $24 \mathrm{~h}$ exhibited $38 \%$ increase in resorufin fluorescence compared to that noted in $\mathrm{m} \varphi$ s incubated in media alone (Fig. $2 \mathrm{~d}, p<0.05$ ). Con-MPs elicited a $26 \%$ decline in JC-1 red/green ratio $(p>0.05)$ and no proliferation in THP-1 m $\varphi$ s. Together, the results presented in Figures 1 and 2 suggested that $T c$ MPs elicited the expression of some of the genes indicative of inflammatory activation in THP- $1 \mathrm{~m} \varphi \mathrm{s}$, and the immune activation was associated with an increase in nitrite release and cell proliferation and a decline in mitochondrial membrane potential in THP-1 m $\varphi s$. The LPS-MPs were more immunostimulatory than the Tc-MPs, demonstrated by the greater induction of proinflammatory gene expression and nitrite release by THP- $1 \mathrm{~m} \varphi s$. 
Fig. 3. Phenotype of MPs induced by Tc infection and chronic Chagas disease. a, b MPs were harvested from in vitro infected human PBMCs (as in Fig. 1, 2). Plasma samples from seropositive chagasic subjects categorized as CA and CS, and $\mathrm{NH}$ controls ( $n=10$ per group) were centrifuged (Materials and Methods) and MPs were harvested. Human MPs were labeled with fluorescence-conjugated antibodies against human molecules and analyzed by flow cytometry. The frequency of surface markers of various cellular origin on MPs isolated from control and Tc-infected PBMCs (a) and chagasic subjects (b) are shown. c C57BL/6 mice were challenged with Tc (10,000 parasites/mouse, $n=5$ mice/group/experiment, 2 experiments), and plasma MPs were collected at day 120 after infection, corresponding to the chronic disease phase. The MPs were labeled with fluorescence-conjugated antibodies against mouse molecules and analyzed by flow cytometry. Bar graphs (mean \pm SEM) show the percent frequency of surface markers of various cellular origin on MPs isolated from media of $T c$-infected PBMCs or the plasma of chronically infected human patients and experimental mice. Significance $(* p<0.05)$ is plotted with respect to normal controls.

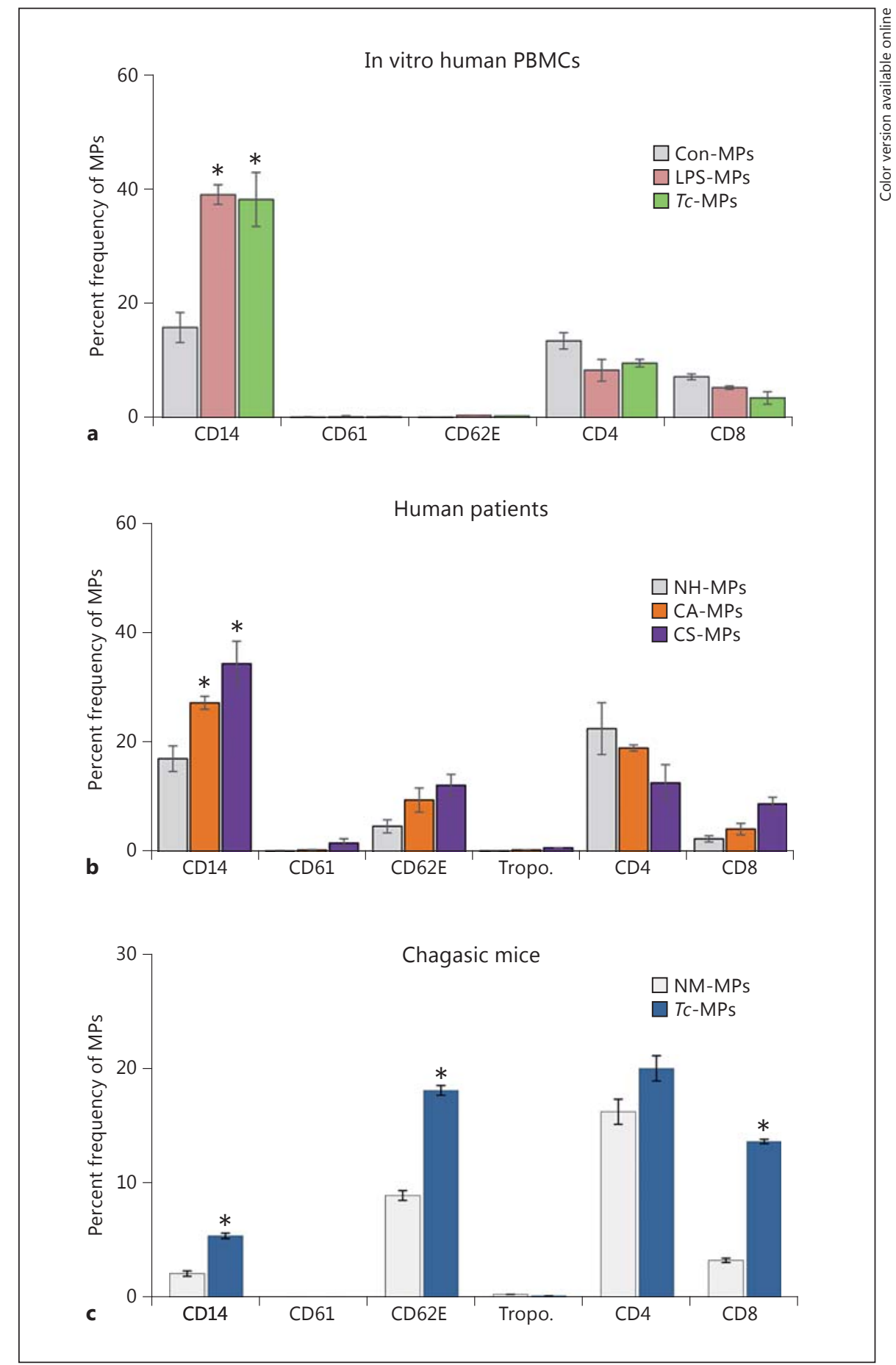

To assess the cellular source of the membranes for MPs released during $T c$ infection, we took a 3-prong approach. Firstly, Tc-MPs were isolated from the supernatants of human PBMCs incubated for $48 \mathrm{~h}$ with $T c$, stained with fluorescence-conjugated antibodies against cell-specific markers, and analyzed by flow cytometry. These data showed that MPs released by human PBMCs upon Tc infection were primarily of monocyte/m $\varphi$ origin (Fig. 3a). Secondly, we examined the phenotype of circulating MPs from chagasic patients. For this, MPs were isolated from 
the plasma of $\mathrm{NH}$ individuals and seropositive subjects characterized as CA and CS for heart disease $(n=10$ per group), and analyzed, as above (Fig. 3b). These data showed that MPs of platelet $\left(\mathrm{CD} 61^{+}\right)$or cardiomyocyte $\left(\right.$ troponin $^{+}$) origin constituted $<2 \%$ of the total circulating MPs in the plasma of normal and chagasic individuals (Fig. 3b). A majority of circulating MPs in the blood of all the human subjects, irrespective of infection and disease status, were of monocyte/ $\mathrm{m} \varphi\left(\mathrm{CD}_{1} 4^{+}\right)$, endothelial $\left(\mathrm{CD}_{2} \mathrm{E}^{+}\right)$, and $\mathrm{CD} 4^{+} / \mathrm{CD}^{+} \mathrm{T}$ lymphocyte origin (CD14 $\geq \mathrm{CD} 4>\mathrm{CD} 62=\mathrm{CD} 8$; Fig. 3b). The chagasic subjects exhibited a substantial increase in the frequency of circulatory MPs that were $\mathrm{CD} 14^{+} / \mathrm{CD} 14^{\text {hi }}$ (up to 2 -fold, $\mathrm{m} \varphi$ marker), $\mathrm{CD} 6 \mathrm{E}^{+} / \mathrm{CD} 6 \mathrm{E}^{\mathrm{hi}}$ (2- to 3-fold, endothelial marker), or $\mathrm{CD}^{+} / \mathrm{CD} 8^{\text {hi }}$ (4.2-fold, $\mathrm{T}$ lymphocytes) (Fig. 3b, $p<0.05$ ). Thirdly, MPs were isolated from the plasma of mice experimentally infected with $T c$, and analyzed by flow cytometry. As noted in human chagasic patients, mice chronically infected with $T c$ exhibited 2.3fold, 2-fold and 4.5-fold increases in circulatory MPs of monocytes/m $\varphi\left(\mathrm{CD}_{14}{ }^{+}\right)$, endothelial $\left(\mathrm{CD}_{2} \mathrm{E}^{+}\right)$, and $\mathrm{CD}^{+} \mathrm{T}$ lymphocyte origin, respectively, compared to that noted in the plasma of normal mice (Fig. $3 c, p<0.05$ ). Together, these results suggested that circulatory MPs originating from activated endothelial cells, $m \varphi s$, and $\mathrm{CD}^{+} \mathrm{T}$ cells were enhanced in chagasic patients and chronically infected mice.

Next, we investigated if circulating MPs present in the plasma of chagasic patients elicited differential THP-1 $\mathrm{m} \varphi$ activation depending upon the clinical disease state. THP-1 $\mathrm{m} \varphi$ s were incubated for $12 \mathrm{~h}$ with MPs isolated from the plasma of $\mathrm{NH}, \mathrm{CA}$, and CS subjects $(n=10$ per group), and total RNA was isolated and reverse-transcribed. The cDNA samples from individuals within a group were pooled into 2 sets, and all samples were analyzed in duplicate by qPCR. The profiling of the gene expression in THP-1m $\varphi$ s using the inflammatory signaling cascade and cytokine/chemokine arrays showed that 34 genes (9 downregulated, 25 upregulated) and 95 genes (16 downregulated, 79 upregulated) were differentially expressed ( $\geq|1.5|$ fold change, $p \leq 0.05)$ by CA-MPs and CS-MPs, respectively, with respect to the MPs of NH controls (online suppl. Table S1). Of these, 20 genes were differentially regulated in THP- $1 \mathrm{~m} \varphi$ s by both CA-MPs and CS-MPs. Furthermore, 14 genes ( $\uparrow$ ADRB2, $\uparrow C C R 3$, TGFBR1, $\uparrow$ NR4A2, $\uparrow$ IL-1B, $\uparrow$ CSF1 $\uparrow C C L 17, \uparrow I L-6 S T$,

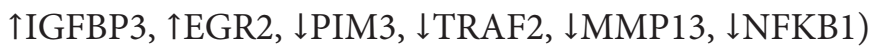
were differentially expressed in a CA-MP-specific manner, and 7 genes ( $\uparrow$ NR3C1, $\uparrow$ IKBKB, $\uparrow$ PLA2G2D, $\uparrow \mathrm{CXCL} 3, \downarrow \mathrm{C} 3, \downarrow \mathrm{NR} 4 \mathrm{~A} 2$, and $\downarrow \mathrm{CD} 14, \geq|2.0|$ fold) were greatly changed in expression in a CS-MPs-specific manner (Fig. 4a, b). Scatter plots show that CA-MPs elicited maximal upregulation of CCR4, EGR2, and CCL3, while CS-MPs elicited maximal upregulation of IKBKB, NR3C1, and TIRAP in THP-1 m $\varphi s$ (Fig. 4c, d). These results suggest that the circulatory MPs from seropositive, $T c$-infected humans elicit a disease-stage-specific inflammatory gene expression profile in THP- $1 \mathrm{~m} \varphi \mathrm{s}$.

To assess the phenotypic effect of human chagasic patients' MPs on $m \varphi s$, we incubated THP-1 $m \varphi s$ for $24 \mathrm{~h}$ with NH-MPs, CA-MPs, and CS-MPs, and evaluated the surface expression of markers of $m \varphi$ activation by flow cytometry and cell viability by alamarBlue assay. THP-1 $m \varphi s$ in vitro incubated with recombinant IFN- $\gamma$, IL-4, and IL-10 cytokines were used as controls. Flow cytometry analysis showed that IFN- $\gamma$-treated (proinflammatory) $\mathrm{m} \varphi$ s were primarily $\mathrm{CD} 80^{+} / \mathrm{CD} 64^{+}$, and IL- $4-$ and IL-10-treated (immunoregulatory) $\mathrm{m} \varphi \mathrm{s}$ were $\mathrm{CD} 163^{+} /$ $\mathrm{CD}^{206}{ }^{+}$. The IL-10-treated THP-1 m $\varphi$ s exhibited a $\mathrm{CD} 16^{\mathrm{hi}} / \mathrm{CD} 200^{\mathrm{hi}}$ profile and the IL-4-treated $\mathrm{m} \varphi$ s exhibited a $\mathrm{CD} 16^{\mathrm{lo}} / \mathrm{CD} 200^{\mathrm{lo}}$ profile [25]. THP-1 $\mathrm{m} \varphi$ s incubated with MPs isolated from the plasma of chagasic patients exhibited no significant change in cell viability and/or cell proliferation (online suppl. Fig. S1A). Furthermore, incubation of THP-1 m $\varphi$ s with CA-MPs resulted in no change in the expression of any of the surface markers indicative of IFN- $\gamma$-induced or IL-4/IL-10-induced phenotypes compared to that noted in $\mathrm{m} \varphi \mathrm{s}$ incubated with NH-MPs (online suppl. Fig. S1B). The CS-MP-induced CD14 ${ }^{+} \mathrm{m} \varphi$ population consisted of a higher number of $\mathrm{CD}^{+} 6^{+}$ $(35 \% \uparrow) / \mathrm{CD} 16^{\mathrm{hi}}(24 \% \uparrow), \mathrm{CD}^{+} 4^{+}(30 \% \uparrow)$, and $\mathrm{CD} 163^{\mathrm{hi}}$ $(10 \% \uparrow) \mathrm{m} \varphi s$ when compared to that noted with CA-MPs. We noted no statistically significant change in the population (as well as intensity) of the $\mathrm{CD} 200^{+}$and $\mathrm{CD} 206^{+} \mathrm{m} \varphi$ population when incubated with MPs from any of the subject group (online suppl. Fig. S1B, e, f). These data suggested that CS-MPs may induce a greater level of inflammatory activation of $\mathrm{m} \varphi \mathrm{s}$ than was induced by CA-MPs, evidenced by increased expression of CD14 and CD16.

We investigated the functional response of $m \varphi s$ to chagasic patients' MPs at 1, 24, and $48 \mathrm{~h}$ after stimulation. The ROS and ${ }^{\circ} \mathrm{NO}$ generation and mitochondrial stress were examined in THP-1 m $\varphi$ s incubated for $1 \mathrm{~h}$ with plasma MPs from NH, CA, and CS human subjects $(n=10$ per group). The THP-1 m $\varphi s$ incubated with CA-MPs or CS-MPs exhibited a 3.8- to 5.6-fold increase in ROS release (Fig. 5a, $p<0.01$ ) and a 2.8 - to 3.1-fold increase in 'NO levels (Fig. 5b, $p<0.001$ ) when compared to that noted in $\mathrm{m} \varphi s$ incubated with $\mathrm{NH}-\mathrm{MPs}$ or media only. Further, THP-1 $m \varphi s$ incubated with CA-MPs and CS- 
Fig. 4. Inflammatory gene expression profile of $m \varphi s$ in response to MPs of chagasic patients. Plasma MPs were isolated from seropositive chagasic subjects categorized as CA and CS, and $\mathrm{NH}$ controls $(n=10$ per group). THP-1 m $\varphi$ s were incubated for $12 \mathrm{~h}$ with human MPs, and gene expression profiling was performed by qRT-PCR using the inflammation signaling cascade and cytokine/chemokine arrays. The differential mRNA level was normalized to housekeeping genes, and fold change in gene expression was calculated in comparison to NH-MP-treated m $\varphi$ s (online suppl. Table $S 1)$. a Venn diagram of differential inflammatory gene expression profile $(\geq|1.5|$ fold change) in m $\varphi s$ incubated with CA-MPs versus CS-MPs. b Mean differential expression of top molecules $(\geq|2.0|$ fold change) induced by CA-MPs and CS-MPs (vs. NH-MPs) in THP-1 m $\varphi s$. c, d Scatter plots show the CA-MP-specific versus CSMP-specific changes in $\mathrm{m} \varphi$ gene expression profile captured by qRT-PCR using the inflammatory signaling cascade (c) and cytokine/chemokine arrays (d). Pink lines mark the $>2$-fold difference in expression (see online version for colors).

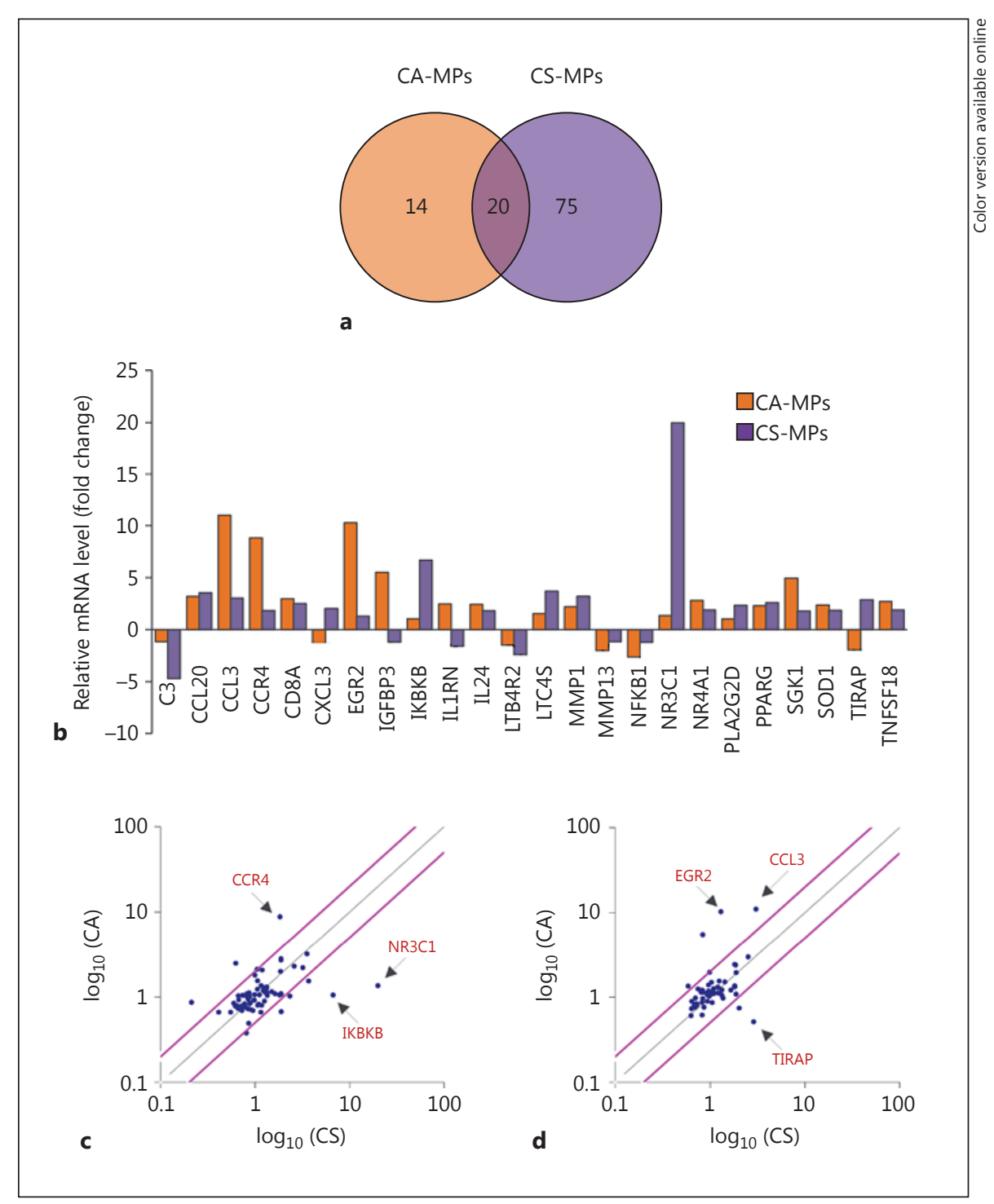

MPs, in comparison to $\mathrm{m} \varphi$ s incubated with media or $\mathrm{NH}$ MPs, exhibited a 33-35\% decline in mitochondrial membrane potential (Fig. 5c, $p<0.01$ ) and a 2.6- to 3 -fold increase in mitochondrial ROS production (Fig. $5 \mathrm{~d}, p<$ 0.001). A Bio-Plex Multiplex Human Cytokine Assay was employed to evaluate the cytokine release in supernatants of THP- $1 \mathrm{~m} \varphi$ s incubated for $24 \mathrm{~h}$ with MPs from $\mathrm{NH}, \mathrm{CA}$, and CS subjects ( $n=10$ per group). These data showed an increase of up to 2 -fold in the release of IL-7 by $m \varphi s$ incubated with CA-MPs and CS-MPs (vs. NH-MPs). The CA-MPs also elicited a 4-fold increase in GCSF levels, while CS-MPs elicited $>2$-fold increase in IL-2 and IL-17 cytokines in THP-1 m $\varphi s$ (Fig. 5e). In another set of experiments, THP-1 m $\varphi$ s were incubated for $48 \mathrm{~h}$ with MPs from $\mathrm{NH}, \mathrm{CA}$, and CS subjects ( $n=10$ per group), and cytokine release in supernatants was evaluated by ELISA. We noted an increase of approximately $20 \%$ in IL- 4 release and no change in IL-10 release in $\mathrm{m} \varphi \mathrm{s}$ incubated with CA-MPs or CS-MPs (vs. NH-MPs; data not shown). Furthermore, THP- $1 \mathrm{~m} \varphi \mathrm{s}$ incubated for $48 \mathrm{~h}$ with CAMPs exhibited a $75 \%$ increase in IL- $1 \beta$ production, and incubation with CS-MPs elicited a $50 \%$ increase in IL- $1 \beta$ and a 2 -fold increase in IFN- $\gamma$ release, respectively, compared to that noted in THP- $1 \mathrm{~m} \varphi s$ incubated with $\mathrm{NH}$ MPs (Fig. 5f, g, $p<0.01$ ). Together, the results presented in Figure 5 suggest that: (a) circulatory MPs from CA and CS subjects elicited oxidative and nitrosative stress and cytokine (i.e., IL-1 $\beta$ and IL-7) release in THP-1 m $\varphi$ s com- 
Fig. 5. Functional activation of $m \varphi s$ by MPs isolated from chagasic patients. MPs were harvested from the plasma of seropositive CA and CS subjects. MPs from seronegative $\mathrm{NH}$ individuals were used as controls. a-d THP-1 m $\varphi$ s were incubated in triplicate with medium only (none) or with $\mathrm{NH}$ MPs, CA-MPs, and CS-MPs for $1 \mathrm{~h}$. Cellfree supernatants were utilized to measure ROS levels by amplex red assay (a) and measure nitrate/nitrite levels by Griess reagent assay (b). c, d THP- $1 \mathrm{~m} \varphi$ s were incubated for $1 \mathrm{~h}$ with MPs as above. Cells were loaded with JC-1 or MitoSOX Red probes, and analyzed by fluorimetry. Changes in mitochondrial membrane potential measured as J aggregates (red)/J monomers (green) ratio (c) and MitoSOX Red fluorescence as a measure of mitochondrial ROS production (d) are shown. e Plasma-derived MPs from $\mathrm{NH}, \mathrm{CA}$, and $\mathrm{CS}$ subjects were added in triplicate to THP-1 m $\varphi s$. Cells were incubated for $24 \mathrm{~h}$, and supernatants were utilized in a Bioplex assay for a panel of 17 human cytokines and chemokines. f, $\mathbf{g}$ ELISA was performed to measure the IL- $1 \beta$ and IFN- $\gamma$ levels in cell-free supernatants collected $48 \mathrm{~h}$ after incubation of THP-1 m $\varphi s$ with MPs. In all bar graphs, data are plotted as mean value \pm SEM, and significance is presented as $* p<$ $0.05,{ }^{* *} p<0.01,{ }^{* * *} p<0.001$ (media only or NH-MPs [controls] vs. CA-MPs or CSMPs).
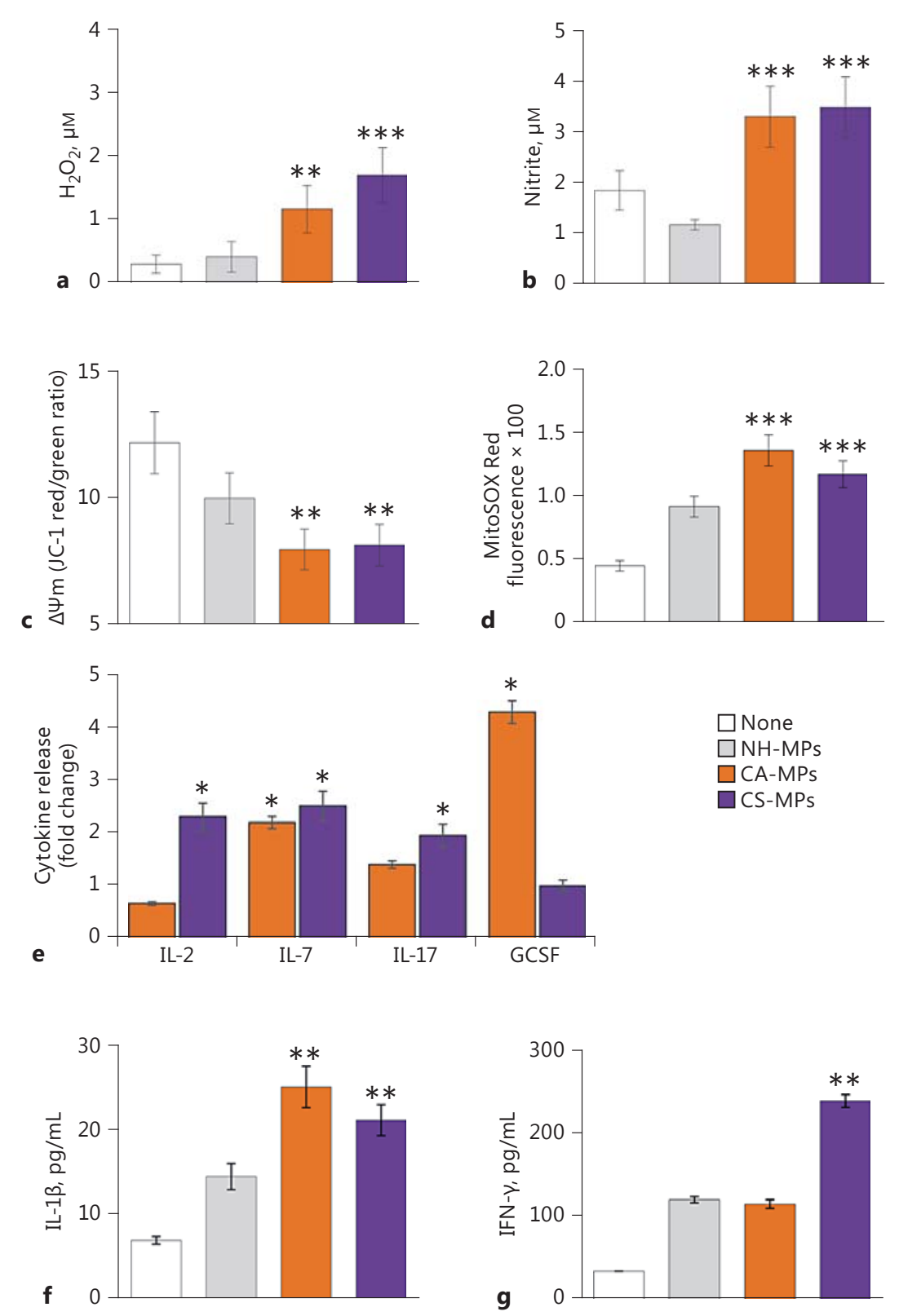

pared to when $\mathrm{m} \varphi \mathrm{s}$ were incubated with media alone or NH-MPs, and (b) GCSF and IL-2/IFN- $\gamma$ are released by THP- $1 \mathrm{~m} \varphi s$ in a CA-MP- and CS-MP-specific manner, respectively.

Finally, we determined if the observed differences in $\mathrm{m} \varphi$ activation by CA-MPs and CS-MPs were reflective of control of the parasite and disease. For this, we utilized an experimental model of vaccination against $T c$ infection.
We have previously shown that a subunit vaccine composed of TcG2 and TcG4 antigens of Tc, delivered by DNA-prime/protein-boost approach, was efficacious in controlling parasite burden, myocarditis, and cardiac remodeling in mice [19]. We isolated MPs from the plasma of nonvaccinated/infected and vaccinated/infected mice at 120 days after infection (corresponding to a chronic disease state) and incubated the MPs with RAW264.7 


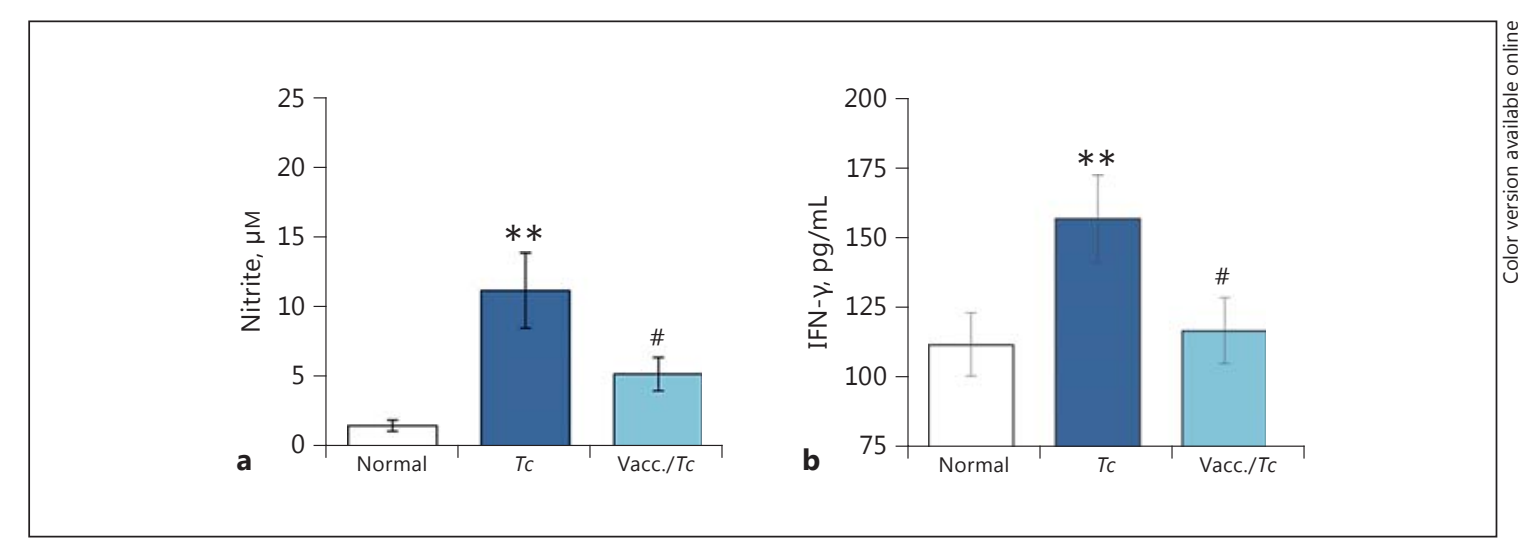

Fig. 6. Nitrite and IFN- $\gamma$ stimulation by MPs from chagasic mice ( \pm anti-Tc vaccine). Mice were vaccinated with TcG2/TcG4 candidate antigens, delivered as DNA-prime/protein-boost vaccine (Materials and Methods). Two weeks after the last immunization, they were infected with Tc. MPs were harvested from the plasma of nonvaccinated/infected and vaccinated/infected mice 120 days after infection. MPs harvested from plasma of nonvaccinated/noninfected (normal) mice were used as controls. RAW264.7 $\mathrm{m} \varphi \mathrm{s}$ were incubated in triplicate for $48 \mathrm{~h}$ with MPs. Supernatants were utilized for measuring the nitrate/nitrite levels by Griess reagent assay (a) and measuring IFN- $\gamma$ levels by ELISA (b). Data are plotted as mean value \pm SEM ( $n=5$ mice/group/experiment, 2 experiments), and significance is presented as ${ }^{* *} p<0.01$ (nonvaccinated/infected vs. normal controls) and ${ }^{\#} p<0.05$ (nonvaccinated/ infected vs. vaccinated/infected). $\mathrm{m} \varphi \mathrm{s}$ for $48 \mathrm{~h}$. Our data showed that MPs isolated from the nonvaccinated/infected mice (vs. MPs from normal mice) elicited a 7.5 -fold and $40 \%$ increase in ${ }^{\circ} \mathrm{NO}$ and IFN- $\gamma$ production, respectively, in RAW264.7 $\mathrm{m} \varphi \mathrm{s}$ (Fig. 6a, b). In comparison, MPs isolated from the plasma of TcG2/ TcG4-vaccinated/infected mice elicited a significantly lower level of $\mathrm{NO}$ release and no IFN- $\gamma$ production (Fig. 6a, b). Together, the results presented in Figure 6 suggest that vaccine-induced control of infection and disease is associated with a significant decline in the ability of MPs to induce the $m \varphi$ activation of the ${ }^{*} \mathrm{NO}$ and IFN- $\gamma$ response.

\section{Discussion}

The currently available invasive tools are not practical for routine screening and monitoring the disease status or for predicting the risk of developing full-blown cardiac failure in $T c$-infected individuals. The cure for chronic patients is routinely determined based upon the conversion to negative serology, which can take up to 8-10 years after treatment $[26,27]$ and occurs in $<15 \%$ of treated adult subjects $[28,29]$. Furthermore, the recently completed BENEFIT clinical trial concluded that trypanocidal therapy with benznidazole in patients with established Chagas cardiomyopathy significantly reduced the parasite detected in the serum but did not reduce cardiac clin- ical deterioration during a 5-year follow-up. These findings affirmed that conversion to negative serology is not synonymous with a cure $[30,31]$. No easy-to-use diagnostic tests for determining a patient's risk of developing CS disease and the efficacy of a treatment in controlling infection or arresting disease progression are available. Therefore, in this study, our goal was to determine whether circulating MPs generated during Tc infection carry the host's signature of inflammatory/oxidative state and provide information regarding clinical disease severity. We utilized an in vitro system, samples from chagasic patients exhibiting different stages of disease development, and a murine model of Tc infection and cure by vaccination, and employed high-throughput transcriptomic and physiological approaches to study the $m \varphi$ response to MPs. Our results suggest that MPs released by human PBMCs infected with Tc in vitro or circulatory MPs present in the plasma of chronically infected chagasic patients and experimentally infected mice were primarily of monocyte/m $\varphi$ and lymphocyte (CD8 > CD4) origin, and exhibited an inflammatory phenotype. This was demonstrated by a decline in mitochondrial membrane potential and an increase in the proinflammatory gene expression profile, and IFN $-\gamma$ and ${ }^{\circ} \mathrm{NO}$ production in $\mathrm{m} \varphi$ s incubated with MPs derived from the 3 models of Tc infection. The key features of the $m \varphi$ response to CS-MPs included a pronounced proinflammatory gene expression profile (Fig. 4), an increase in the expression of the NR3C1, 
IKBKB, and TIRAP genes, and substantially higher levels of IFN- $\gamma$ release, while the $m \varphi$ response to CA-MPs was captured by increased expression of CCR4, EGR2, and CCL3, and GCSF production. Furthermore, vaccinated mice, which were previously shown to control parasite persistence and chronic myocarditis $[19,20]$, produced MPs that elicited no ${ }^{\circ} \mathrm{NO}$ and less IFN- $\gamma$ in $\mathrm{m} \varphi$ s than was noted with MPs of nonvaccinated/infected mice. To the best of our knowledge, this is the first study demonstrating that circulating MPs predict the in vivo levels of the oxidative and inflammatory state and have potential utility in evaluating disease severity and the efficacy of vaccines and drug therapies against CCM.

Several Tc-derived molecules (e.g., glycosylphosphatidylinositols, mucin-like glycoproteins) act as TLR2 and TLR4 agonists and induce the production of ${ }^{\circ} \mathrm{NO}$ and inflammatory cytokines and chemokines via cells of the monocytic lineage [review 32]. Other study groups have shown that TLR $4^{-/-} \mathrm{m} \varphi$ s are deficient in the production of trypanocidal ${ }^{\circ} \mathrm{NO}$ and ROS and fail to control parasite

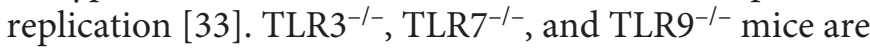
also susceptible to $T c$ infection [34], and it has been suggested that $T c$-DNA-dependent TLR/Myd88 plays an important role in bridging innate to acquired immunity in the context of the control of $T c$ infection. These studies support the role of innate immune cells (e.g., $\mathrm{m} \varphi s$, dendritic cells) in regulating $T c$ infection.

Indeed, the host is capable of controlling the acute parasitemia to barely detectable levels. Why the chronic inflammation then persists in the host is not completely understood. Our results in this study provide some clues as to the source of the stimulus contributing to the persistence of the inflammatory infiltrate in Chagas disease. We propose that after controlling the acute infection, the clearance of activated immune cells is necessary to maintain the homeostatic state. During this process, membranes shed by activated immune cells produce circulatory MPs and these MPs may potentially serve as damageassociated molecular patterns and activate immune cells by engaging TLRs and NLRs. This notion is supported by the observation that circulating MPs in chagasic patients and in the experimentally infected mice were composed of membranes shed by endothelial cells, $\mathrm{m} \varphi \mathrm{s}$, and T lymphocytes, all of which are known to be activated during infection, and which contribute to the chronic inflammatory pathology in Chagas disease [reviews 35, 36]. Furthermore, the MPs present in the plasma of chagasic humans and experimentally infected mice were capable of signaling a proinflammatory phenotype in the $\mathrm{m} \varphi \mathrm{s}$, demonstrated by increased proliferation, ${ }^{\circ} \mathrm{NO}$ release, inflam- matory gene expression, and cytokine (IL- $1 \beta$, IFN- $\gamma$ ) production. The IL-12 and IL-18 cytokines are shown to induce IFN- $\gamma$ in $m \varphi s$ [37], and the latter can contribute to the proinflammatory activation of $\mathrm{m} \varphi \mathrm{s}$ in an autocrine manner [38]. Additional studies evaluating the proteomic and functional profile of circulating MPs will provide insights into the pathological mechanisms that may contribute to generating MPs; however, our results provide a strong indication that the proinflammatory nature of the circulating MPs may, at least partially, contribute to the persistence of chronic inflammation during Chagas disease.

We have previously shown that the incubation of $m \varphi s$ with sera of chagasic mice elicited a proinflammatory phenotype (CD64 ${ }^{\text {hi }} \mathrm{CD} 80^{\text {hi }}$ ) and functional response (increased TNF- $\alpha / \mathrm{IFN}-\gamma$ production) [25]. In this study, circulatory MPs from chagasic patients and experimentally infected mice produced a similar proinflammatory activation of $m \varphi s$ (Fig. 4-6), noted when $m \varphi s$ were incubated with complete sera or plasma of chagasic mice [25]. Incubation with MP-free sera or plasma from chagasic mice or patients elicited no response in $\mathrm{m} \varphi s$ (data not shown). These results suggest that it is MPs, and not the soluble molecules present in the systemic circulation, that carry the proinflammatory signature of Chagas disease.

$T c$ infection mobilizes innate and adaptive immune responses that induce $m \varphi$ activation and keep infection under control [39]. Experimental animals and humans elicit potent adaptive $\mathrm{B}$ and $\mathrm{T}$ cell immunity to $T c$ infection, and are capable of controlling the acute circulating and tissue parasite burden $[35,36,40]$. We showed that the MPs isolated from clinically symptomatic chagasic patients and experimentally infected mice elicited a substantial increase in cytokines (IL-1 $\beta$, IL-7, and IFN- $\gamma$ ), ROS, and $\mathrm{NO}$ production in $\mathrm{m} \varphi \mathrm{s}$ (Fig. 5,6 ). These results suggest that circulatory MPs constitute a pathomechanism in chronic Chagas disease, and the therapies capable of preventing cellular injury (i.e., inhibiting the generation of MPs) or reprogramming the $m \varphi s$ (i.e., inhibiting proinflammatory cytokines, ROS, and ${ }^{\circ} \mathrm{NO}$ production) will be beneficial in halting the feedback cycle of $\mathrm{m} \varphi$ activation and the persistence of pathological inflammatory stress in Chagas disease. This notion is strongly supported by our findings that complete sera or MPs harvested from nonvaccinated/infected (vs. vaccinated/ infected) mice elicited a pronounced IFN- $\gamma$ and ${ }^{-N O}$ production response in $m \varphi s$ (Fig. 6) [25]. Likewise, the MPs of seropositive individuals who exhibited clinical disease induced a more robust proinflammatory gene expression profile, cytokine release (IL- $1 \beta$, IL- 2 , and IFN- $\gamma$ ), and mi- 
totoxic phenotype, while the MPs of seropositive individuals who had not yet developed clinical disease elicited a mild-to-moderate gene expression profile and no IFN- $\gamma$ in THP-1 m $\varphi s$ (Fig. 4, 5).

'NO release was suppressed in $m \varphi s$ stimulated with MPs from vaccinated/infected mice compared to the MPs from nonvaccinated/noninfected mice; however, we did not observe this trend with the MPs from CA versus CS patients, as the MPs from both groups elevated $\mathrm{NO}$ release from THP-1 m $\varphi s$. We speculate that the difference in the RAW264.7 versus THP- $1 \mathrm{~m} \varphi \mathrm{s}^{\prime}$ ability to respond to mouse versus human MPs, and the difference in the composition of MPs in mice and in humans, may contribute to the outcome that we observed, with respect to the control of ${ }^{*} \mathrm{NO}$ release when MPs from infected/vaccinated mice and CA humans were used. Resistance to $T c$ infection in humans and in mice may vary according to the genetic background of the host and the virulence of the parasite strain, and contribute to differential ${ }^{\circ} \mathrm{NO}$ production as well.

In summary, we used an in vitro system, an experimental model of vaccination, and human chagasic patients, and showed that MPs of monocyte/m $\varphi$ and lymphocyte origin are produced during the course of $T c$ infection and chronic disease progression, and that these MPs elicit proinflammatory activation of $m \varphi s$. Our results suggest that MP-induced activation of a differential inflammatory gene expression profile, cytokine release, and $\mathrm{NO}$ production in $\mathrm{m} \varphi \mathrm{s}$ reflects the severity of the disease state in the chagasic host. These results give impetus for appraising the MP signature of a large number of individuals who exhibit varying degree of CCM sever- ity. We hope that our MPs and in vitro cell-based assays can have the potential utility and power for identifying the risk of clinical disease development and evaluating the efficacy of therapies for controlling Chagas disease.

\section{Acknowledgements}

We appreciate the consistent and long-standing support of Dr. Ines Vidal (biochemist of the Central Laboratory of San Bernardo Hospital, Salta, Argentina) and Federico Ramos and Alejandro Uncos (lab technicians) in sample collection and processing and serological testing. In addition, we thank Yolanda Huertas, Rosa Rodas, and Liliana Villagran (nurses and office secretary of the Cardiology Unit at San Bernardo Hospital, Salta, Argentina) for their help in patient recruitment and maintenance of clinical records. The funders had no role in study design, data collection and analysis, decision to publish, or preparation of the paper.

\section{Funding Sources}

This work was supported in part by grants from the National Institutes of Health, National Institute of Allergy and Infectious Diseases (R01 AI054578 and R21 AI107227), and National Heart Lung Blood Institute (R01 HL094802) to N.J.G. L.S. and S.G. received a fellowship from the Summer Undergraduate Research Program and Sealy Center for Vaccine Development, respectively, at the UTMB Galveston. S.K. was awarded McLaughlin Endowment (UTMB) and American Heart Association pre-doc fellowships. M.P.Z. was supported by the Consejo Nacional de Investigaciones Científicas y Técnicas (CONICET), Argentina.

\section{Disclosure Statement}

The authors have no competing interests.

\section{References}

1 World Health Organization: Chagas disease: control and elimination; in: Report of the Secretariat WHO, Geneva, 2010. UNDP/World Bank/WHO. http://apps.who.int/gb/ebwha/ pdf_files/WHA63/A63_17-en.pdf.

2 Bern C, Montgomery SP: An estimate of the burden of Chagas disease in the United States. Clin Infect Dis 2009;49:e52-e54.

3 Tanowitz HB, Machado FS, Spray DC, Friedman JM, Weiss OS, Lora J, Nascimento D, Nunes MC, Garg NJ, Ribeiro AL: Developments in the manangement of chagasic cardiomyopathy. Expert Rev Cardiovasc Ther 2016;13:1393-1409.
4 Munoz-Fernandez MA, Fernandez MA, Fresno M: Activation of human macrophages for the killing of intracellular Trypanosoma cruzi by TNF-alpha and IFN-gamma through a nitric oxide-dependent mechanism. Immunol Lett 1992;33:35-40.

5 Plasman N, Metz G, Vray B: Interferon-gamma-activated immature macrophages exhibit a high Trypanosoma cruzi infection rate associated with a low production of both nitric oxide and tumor necrosis factor-alpha. Parasitol Res 1994;80:554-558.

6 Alvarez MN, Peluffo G, Piacenza L, Radi R: Intraphagosomal peroxynitrite as a macrophagederived cytotoxin against internalized Trypanosoma cruzi: consequences for oxidative killing and role of microbial peroxiredoxins in infectivity. J Biol Chem 2011;286:6627-6640.
7 Cardoso MS, Reis-Cunha JL, Bartholomeu DC: Evasion of the immune response by Trypanosoma cruzi during acute infection. Front Immunol 2015;6:659.

8 Zhang L, Tarleton RL: Parasite persistence correlates with disease severity and localization in chronic Chagas disease. J Infect Dis 1999; 180:480-486.

9 Wen JJ, Dhiman M, Whorton EB, Garg NJ Tissue-specific oxidative imbalance and mitochondrial dysfunction during Trypanosoma cruzi infection in mice. Microbes Infect 2008;10:1201-1209. 
10 Wen J-J, Bhatia V, Popov VL, Garg NJ: Phenyl-alpha-tert-butyl nitrone reverses mitochondrial decay in acute Chagas disease. Am J Pathol 2006;169:1953-1964.

11 Wen J-J, Gupta S, Guan Z, Dhiman M, Condon D, Lui CY, Garg NJ: Phenyl-alpha-tertbutyl-nitrone and benzonidazole treatment controlled the mitochondrial oxidative stress and evolution of cardiomyopathy in chronic chagasic rats. J Am Coll Cardiol 2010;55: 2499-2508.

12 Dhiman M, Wan X-X, Popov VL, Vargas G, Garg NJ: MnSOD ${ }^{\text {tg }}$ mice control myocardial inflammatory and oxidative stress and remodeling responses elicited in chronic Chagas disease. J Am Heart Assoc 2013;2:e000302.

13 Dhiman M, Garg NJ: NADPH oxidase inhibition ameliorates Trypanosoma cruzi-induced myocarditis during Chagas disease. J Pathol 2011;225:583-596.

14 Dhiman M, Garg NJ: P47phox-/- mice are compromised in expansion and activation of CD8+ T cells and susceptible to Trypanosoma cruzi infection. PLoS Pathog 2014;10: e1004516.

15 Dhiman M, Zago MP, Nunez S, Nunez-Burgio F, Garg NJ: Cardiac oxidized antigens are targets of immune recognition by antibodies and potential molecular determinants in Chagas disease pathogenesis. PLoS One 2012;7: e28449.

16 Burnouf T, Chou ML, Goubran H, Cognasse F, Garraud O, Seghatchian J: An overview of the role of microparticles/microvesicles in blood components: are they clinically beneficial or harmful? Transfus Apher Sci 2015;53: 137-145.

17 Shantsila E, Kamphuisen PW, Lip GY: Circulating microparticles in cardiovascular disease: implications for atherogenesis and atherothrombosis. J Thromb Haemost 2010;8: 2358-2368.

18 Wen JJ, Zago MP, Nunez S, Gupta S, Burgos FN, Garg NJ: Serum proteomic signature of human chagasic patients for the identification of novel protein biomarkers of disease. Mol Cell Proteomics 2012;11:435-452.

19 Gupta S, Garg NJ: Prophylactic efficacy of TcVac2R against Trypanosoma cruzi in mice. PLoS Negl Trop Dis 2010;4:e797.

20 Gupta S, Garg NJ: A two-component DNAprime/protein-boost vaccination strategy for eliciting long-term, protective $\mathrm{T}$ cell immunity against Trypanosoma cruzi. PLoS Pathog 2015;11:e1004828.

21 Dey N, Sinha M, Gupta S, Gonzalez MN, Fang R, Endsley JJ, Luxon BA, Garg NJ: Caspase-1/ ASC inflammasome-mediated activation of
IL-1 $\beta$-ROS-NF- $\kappa B$ pathway for control of Trypanosoma cruzi replication and survival is dispensable in NLRP3-/- macrophages. PLoS One 2014;9:e111539.

22 Robertson RC, Guiheneuf F, Bahar B, Schmid M, Stengel DB, Fitzgerald GF, Ross RP, Stanton C: The anti-inflammatory effect of algaederived lipid extracts on lipopolysaccharide (LPS)-stimulated human THP-1 macrophages. Mar Drugs 2015;13:5402-5424.

23 Crean D, Cummins EP, Bahar B, Mohan H, McMorrow JP, Murphy EP: Adenosine modulates NR4A orphan nuclear receptors to attenuate hyperinflammatory responses in monocytic cells. J Immunol 2015;195:14361448 .

24 Kleinbongard P, Rassaf T, Dejam A, Kerber S, Kelm M: Griess method for nitrite measurement of aqueous and protein-containing samples. Methods Enzymol 2002;359:158168.

25 Gupta S, Silva TS, Osizugbo JE, Tucker L, Spratt HM, Garg NJ: Serum-mediated activation of macrophages reflects $\mathrm{TcVac} 2$ vaccine efficacy against Chagas disease. Infect Immun 2014;82:1382-1389.

26 Cancado JR: Long-term evaluation of etiological treatment of Chagas disease with benznidazole. Rev Inst Med Trop Sao Paulo 2002;44: 29-37.

27 Cancado JR: Criteria of Chagas disease cure. Mem Inst Oswaldo Cruz 1999;94(suppl 1): 331-335.

28 Viotti R, Vigliano C, Lococo B, Bertocci G, Petti M, Alvarez MG, Postan M, Armenti $\mathrm{AH}$ : Long-term cardiac outcome of treating chronic Chagas disease with benznidazole versus no treatment: a nonrandomized trial. Ann Intern Med 2006;144:724-734.

29 Fabbro DL, Streiger ML, Arias ED, Bizai ML, del Barco M, Amicone NA: Trypanocide treatment among adults with chronic Chagas disease living in Santa Fe City (Argentina), over a mean follow-up of 21 years: parasitological, serological and clinical evolution. Rev Soc Bras Med Trop 2007;40:1-10.

30 Bautista-Lopez NL, Morillo CA, Lopez-Jaramillo P, Quiroz R, Luengas C, Silva SY, Galipeau J, Lalu MM, Schulz R: Matrix metalloproteinases 2 and 9 as diagnostic markers in the progression to Chagas cardiomyopathy. Am Heart J 2013;165:558-566.

31 Morillo CA, Marin-Neto JA, Avezum A, Sosa-Estani S, Rassi A Jr, Rosas F, Villena E, Quiroz R, Bonilla R, Britto C, et al: Randomized trial of benznidazole for chronic Chagas' cardiomyopathy. N Engl J Med 2015;373: 1295-1306.
32 Rodrigues MM, Oliveira AC, Bellio M: The immune response to Trypanosoma cruzi: role of toll-like receptors and perspectives for vaccine development. J Parasitol Res 2012;2012: 507874.

33 Oliveira AC, de Alencar BC, Tzelepis F, Klezewsky W, da Silva RN, Neves FS, Cavalcanti GS, Boscardin S, Nunes MP, Santiago $\mathrm{MF}$, et al: Impaired innate immunity in TLR4(-/-) mice but preserved CD8+ T cell responses against Trypanosoma cruzi in TLR4-, TLR2-, TLR9- or Myd88-deficient mice. PLoS Pathog 2010;6:e1000870.

34 Caetano BC, Carmo BB, Melo MB, Cerny A, dos Santos SL, Bartholomeu DC, Golenbock DT, Gazzinelli RT: Requirement of UNC93B1 reveals a critical role for TLR7 in host resistance to primary infection with Trypanosoma cruzi. J Immunol 2011;187:1903-1911.

35 Machado FS, Dutra WO, Esper L, Gollob KJ, Teixeira MM, Weiss LM, Nagajyothi F, Tanowitz HB, Garg NJ: Current understanding of immunity to Trypanosoma cruzi infection and pathogenesis of Chagas disease. Semin Immunopathol 2012;34:753770 .

36 Tanowitz HB, Wen JJ, Machado FS, Desruisseaux MS, Robello C, Garg NJ: Trypanosoma cruzi and Chagas disease: innate immunity, ROS, and cardiovascular system; in Gavins NE, Stokes KY (eds): Vascular Responses to Pathogens. Waltham, Academic Press/Elsevier, 2016, pp 183-193.

37 Darwich L, Coma G, Pena R, Bellido R, Blanco EJ, Este JA, Borras FE, Clotet B, Ruiz L, Rosell A, et al: Secretion of interferon-gamma by human macrophages demonstrated at the single-cell level after costimulation with interleukin (IL)-12 plus IL-18. Immunology 2009;126:386-393.

38 Munder M, Mallo M, Eichmann K, Modolell M: Murine macrophages secrete interferon gamma upon combined stimulation with interleukin (IL)-12 and IL-18: a novel pathway of autocrine macrophage activation. J Exp Med 1998;187:2103-2108.

39 Ribeiro-Gomes FL, Lopes MF, DosReis GA: Negative Signaling and Modulation of Macrophage Function in Trypanosoma cruzi Infection. Madame Curie Bioscience Database, edited by NCBI Bookshelf. Austin, Landes Bioscience, 2000.

40 Nagajyothi F, Machado FS, Burleigh BA, Jelicks LA, Scherer PE, Mukherjee S, Lisanti MP, Weiss LM, Garg NJ, Tanowitz HB: Mechanisms of Trypanosoma cruzi persistence in Chagas disease. Cell Microbiol 2012; 14:634-643. 